\title{
Perceptual and performed accents in musical sequences
}

\author{
CAROLYN DRAKE \\ CNRS URA316, Université René Descartes, Paris, France
}

(Claude Bonnet, Sponsor)

\begin{abstract}
Some events in musical sequences are not performed as indicated in the score, but rather undergo systematic timing and intensity variations (played louder/softer and longer/shorter). Traditionally, these performance variations are considered to be related to a musician's desire to transmit a particular musical interpretation to the listener. We suggest an alternative perceptual explanation that considers that the performance variations may result from constraints of the auditory system, with some events played louder and/or longer because they are heard softer and/or shorter. Two experiments are presented to distinguish between these two explanations. Subjects heard short musical sequences containing rhythmic grouping and melodic accents. Their task was to detect and identify the position of one event that was either slightly longer/shorter (Experiment 1) or louder/softer (Experiment 2) than other events in the sequence. Sensitivity to intensity changes was unaffected by the physical characteristics of the events in the sequences, but sensitivity to duration changes varied in relation to both rhythmic grouping and melodic accents. Thus, these results provide support for the perceptual hypothesis in the case of timing changes, but not for intensity changes.
\end{abstract}

Music-performance studies demonstrate that pianists rarely perform a piece of music exactly as written in the musical score: notes are played longer or shorter, louder or softer than indicated. These performance variations are frequently equated with the performers' intent to convey a particular musical message or expressive interpretation to the listener. This paper investigates the proposition that some performance variations may result from more fundamental mechanisms related to constraints of the auditory system that influence the way both the listener and the performer perceive a sequence of auditory events.

\section{Performance Variations}

Timing and intensity variations recorded during music performances are not just random variations due to problems of motor control or other restraints, but are related to multiple levels of musical structure (Clarke, 1988; Drake, 1990; Drake \& Palmer, in press; Palmer, 1989; Seashore, 1938; Shaffer, Clarke, \& Todd, 1985; Sloboda, 1983). For instance, in a musical piece which can be subdivided into musical phrases, interonset intervals (IOI, the time in milliseconds between the onset of one note and the onset of the following note) are directly related to the position of the note in the phrase, with a gradual lengthening of notes toward the end of the phrase (Palmer, 1989;

This work was carried out at Ohio State University while the author was on a Postdoctoral Fellowship offered by that University. I wish to thank Mari Jones and Caroline Palmer for their advice and support and Marie-Claire Botte for her comments on a previous draft of this manuscript. Correspondence should be addressed to C. Drake, Laboratoire de Psychologie Experimentale, 28, rue Serpente, 75006 Paris, France.
Todd, 1985). This phrase-final lengthening varies with the performers' interpretation of the music, and it is less pronounced in mechanical performances. The traditional explanation is that this type of performance variation is related to the performer's desire to convey a particular musical interpretation to the listener. It will be referred to as the musical expression hypothesis.

Figure 1 presents examples of systematic variations related to specific combinations of notes at a lower hierarchical level within a musical phrase (Drake \& Palmer, in press; Shaffer, Clarke, \& Todd, 1985). A first set is related to rhythmic groups: a long note following a series of short notes is performed louder than surrounding events and is delayed in onset (preceded by a longer interval). A second set is related to melodic contour: events on melodic jumps and turns are performed louder than surrounding events and delayed in onset (preceded by a longer interval). Traditionally, the musical expression hypothesis is also applied to these lower level performance variations: performers emphasize events that they consider important in order to draw the listeners' attention to them, thus conveying their particular musical interpretation.

However, the musical expression hypothesis has several limitations: if variations are related to the performers' wish to convey a particular musical interpretation to the listener, then they should be under the performers' voluntary control, that is, they should vary with the musical context and disappear if the musician so desires. However, when pianists were asked to perform simple musical sequences containing instances of rhythmic groups and melodic jumps and turns in either a musical or a mechanical fashion (without adding any musical expression), the 
written in score

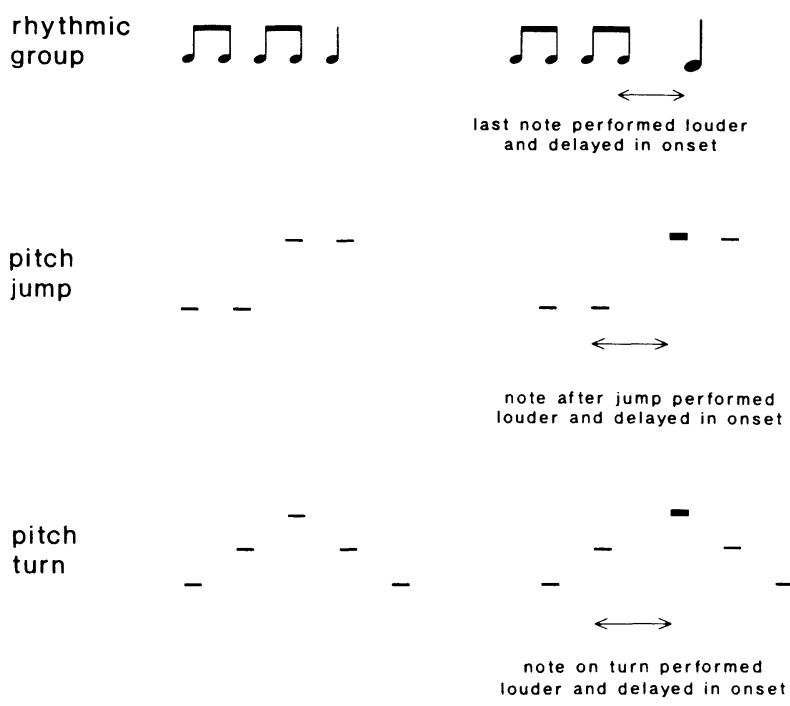

Figure 1. Examples of performance variations.

intensity variations disappeared in the mechanical performances, whereas the timing variations hardly decreased at all (Drake \& Palmer, in press). A second source of evidence against the musical expression hypothesis in the case of low level variations was observed when adult musicians and nonmusicians and 5- and 7-year-old children were asked to reproduce short musical rhythms. The same timing variations were observed for all groups of subjects (even the 5-year-old children), whereas the intensity variations varied between the adult musicians and nonmusicians and were absent for the children (Drake, 1990). Thus, these two types of comparisons indicate that the timing and intensity variations result from distinct processes and that the musical expression hypothesis cannot satisfactorily explain all observed variations.

\section{An Explanation Based on Auditory Constraints}

An alternative explanation is that performance variations may originate in purely perceptual phenomena, with the auditory system actually "distorting"' the perception of some physical characteristics of auditory events embedded in a complex sequence: some events may be played louder/softer or longer/shorter than other events to compensate for hearing them softer/louder or shorter/longer. This explanation will be referred to as the perceptual hypothesis. Several perception studies have indicated that the physical characteristics of a sequence do influence the ability to detect temporal changes within that sequence (Halpern \& Darwin, 1982; Hirsh, Monahan, Grant, \& Singh, 1990; Monahan \& Hirsh, 1990), although it is difficult to apply these findings to musical contexts. Little is known about the ability to detect intensity changes of events in sequences.

The two studies presented here use a purely perceptual task to investigate the ability to detect and identify the po- sition of both timing and intensity variations in short, music-like stimuli in order to test the validity of the perceptual hypothesis. This hypothesis predicts that certain duration and pitch changes in the sequences will lead to variations in the sensitivity to timing and intensity variations. Subjects heard a short sequence of tones in which one interval was longer/shorter than the others (Experiment 1) or in which one event was louder/softer than the others (Experiment 2). Their task was to indicate whether or not they detected a timing/intensity change and to identify its position. Support for the perceptual hypothesis will be obtained if sensitivity varies as a function of the physical characteristics of the sequence.

\section{EXPERIMENT 1 Timing}

\section{Method}

Subjects. Seven groups of 8 undergraduate students at Ohio State University took part in this study and received credit for their participation. They were all nonmusicians with no more than 1 year of musical training.

Stimuli. Figure 2 presents the seven sequences of six sine tones used in this experiment: (1) two isochronous sequences with IOIs of $300 \mathrm{msec}$ and $600 \mathrm{msec}$, respectively; (2) three rhythmic sequences containing four short IOIs $(300 \mathrm{msec})$ and one long IOI $(600 \mathrm{msec}$; the last IOI is always long) that only vary by the order of events; and (3) two melodic sequences with IOIs of $300 \mathrm{msec}$, one with pitch jumps and one with a pitch turn. Onset to offset of all events was $50 \mathrm{msec}$, with $10-\mathrm{msec}$ rise and fall times. Each interval was increased or decreased in duration by $0 \%, 6.7 \%, 10 \%, 13.3 \%$, or $16.7 \%$. All tones were $80 \mathrm{~dB}$ SPL and a C4 $(440 \mathrm{~Hz})$. The stimuli were presented binaurally over AKG Model K240 earphones.

Procedure. The subjects' task was to indicate which of the five intervals in the sequence seemed longer/shorter than the others. Alternatively, they could say that all the intervals were the same duration. For the rhythmic sequences, it was explained that some of the intervals were twice the length of the others and that the subjects' task was to ignore this and

\begin{tabular}{|c|c|c|c|c|c|c|}
\hline rapid isochronous & 1 & 1 & 1 & 1 & 1 & 1 \\
\hline slow isochronous & I & & I & & 1 & \\
\hline rhythm 1 & 1 & 1 & I & & 1 & 1 \\
\hline rhythm 2 & 1 & I & 1 & I & I & \\
\hline rhythm 3 & 1 & & I & I & I & I \\
\hline
\end{tabular}

jumps

$$
\underline{c} \underline{\mathrm{c}} \underline{\mathrm{c}}
$$

turns

$$
\underline{d}^{\underline{e}} \underline{f}^{e} \underline{d}
$$

Figure 2. Stimuli used in Experiments 1 and 2. 
detect smaller timing variations from this pattern. This was easily understood after two trial sequences. Each block contained trials with the five percentage increments on each of the five intervals ( 25 trials). The trials containing increased $(+)$ and decreased $(-)$ intervals were blocked separately, with the subjects doing three sets of one and then three sets of the other, or visa versa. Each set lasted about $8 \mathrm{~min}$, and there was a 2-min pause between sets. So, in all, the subjects heard one sequence 150 times. The subjects were randomly assigned to one of the seven sequences and to one of the two counterbalanced orders $(+1-$ or $-1+)$.

Dependent measures. The data were analyzed in two ways: (1) the percentage of detections, that is, how frequently the subject correctly detected a difference, whether or not he/she correctly identified the position of this change, and (2) the percentage of correct identifications, that is, how frequently the subject correctly identified the position of the change. In addition, as an indication of the size of the observed ef fects, we calculated the percentage change of interval duration correctly detected $50 \%$ of the time.

\section{Results}

Analyses of variance (ANOVAs) were carried out for each sequence on the percentage of detections and correct identifications of the position of change. These ANOVAs were by position in sequence (5), direction of change (increase or decrease), trial (3), and order $(+/-$ or $-1+$ ). The only significant main effects and interactions were related to position.

Isochronous sequences. For both the slow and rapid sequences, duration changes were detected and identified equally well in all positions. On average, changes were detected correctly on $78 \%$ of trials, and the position was identified correctly on $61 \%$ of trials. Average detection thresholds were $6.9 \%$, and average identification thresholds were $11 \%$.

Rhythmic sequences. Figure 3 shows the percentage of detections of temporal increases and decreases for one of the three rhythmic sequences (typical of the others) and for each position within the sequence. The perceptual hypothesis predicts that sensitivity to temporal changes will be lower in the interval (marked by a box in Figure 3) that precedes the long interval than in the other intervals. Main position effects were seen for the three rhythms [R1, $F(4,24)=5.9, p<.01 ; \mathrm{R} 2, F(4,24)=7.3, p<.01$; R3, $F(4,24)=5, p<.01$ ], and planned comparisons comparing each short interval before a long interval with the short intervals preceding it revealed significant effects

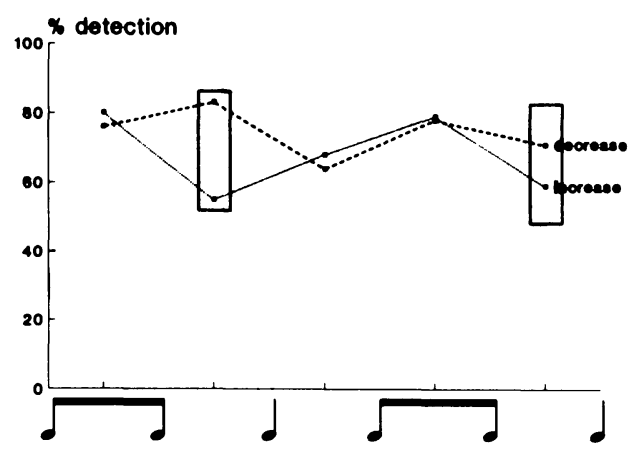

Figure 3. Experiment 1: Percentage of detections for increased and decreased durations in one of the rhythmic sequences. Lower sensitivity is predicted in the intervals contained in the boxes.

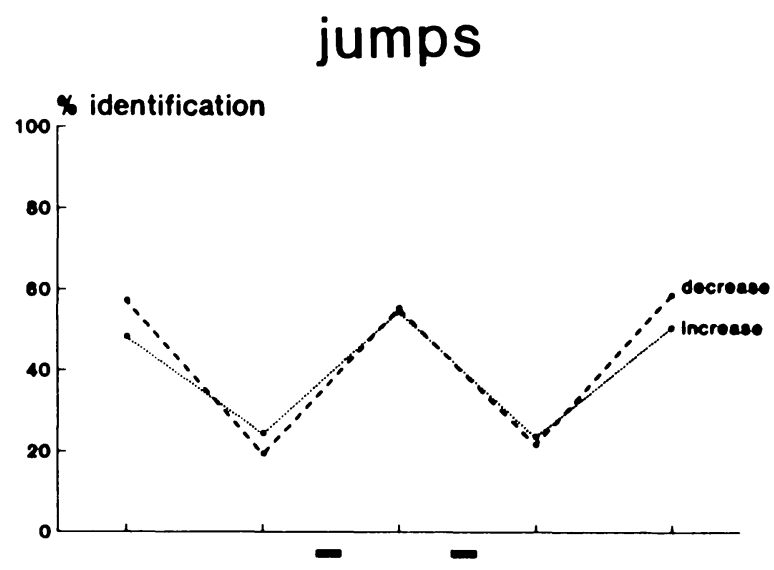

Figure 4. Experiment 1: Percentage of identifications for increased and decreased durations in the melodic jumps.

for all the increases but not for the decreases. Similar patterns were seen for the identifications.

Melodic sequences. The perceptual hypothesis predicts that sensitivity to changes in duration of intervals before and after jumps and turns will be lower than for changes in duration of other intervals. Melodic jumps exist at Positions 3 and 5. Figure 4 shows the percentage of identifications of increases and decreases for the melodic jumps. The expected pattern was not seen for the detection data, but it was for the identification data, where there was a main position effect $[F(4,24)=6.7, p<.01]$ : changes in Positions 2 and 4 were much harder to identify than were changes in Positions 1, 3, and 5 [P1, P3, and $\mathrm{P} 5$ vs. $\mathrm{P} 2$ and $\mathrm{P} 4, F(1,6)=16.3, p<.01]$. No changes in sensitivity were seen in relation to the melodic turn.

\section{Discussion}

So, sensitivity to temporal changes in short sequences is affected by the physical characteristics of the sequence: it is much harder to detect and identify a decrease than an increase in the duration of the interval preceding a long interval, and, similarly, it is harder to identify the position of a temporal change before a jump. These two results provide support for the perceptual hypothesis. However, no such pattern was observed for the melodic turns.

\section{EXPERIMENT 2 Intensity}

\section{Method}

Subjects. Seven groups of 10 undergraduate students were selected as in Experiment 1.

Stimuli. The same seven sequences from Experiment 1 were used here. Procedure. The subjects' task was to indicate which of the six tones in the sequence seemed louder or softer than the others. Alternatively, they could say that all the intervals were the same loudness. In each block, there were five possible increments $(0, .75,1.5,2.25$, and $3 \mathrm{~dB})$ on six possible tones, making a total of 30 trials. Other details are the same as in Experiment 1. 


\section{jumps}

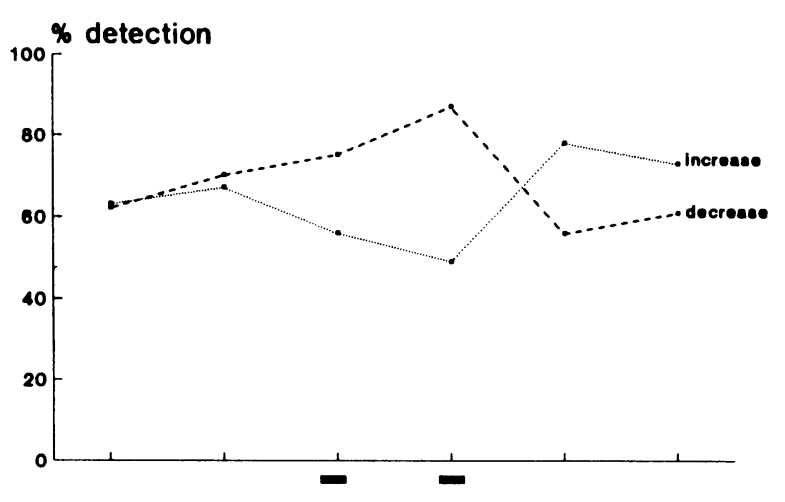

Figure 5. Experiment 2: Percentage of detections for increased and decreased intensities in the melodic jumps.

\section{Results}

Isochronous sequences. No systematic differences were observed as a function of the position in the sequence for either the slow or rapid isochronous sequences. On average, changes were detected correctly on $75 \%$ of trials, and the position was identified correctly on $65 \%$ of trials. Average detection thresholds were about $1.5 \mathrm{~dB}$, and average identification thresholds were about $2 \mathrm{~dB}$.

Rhythmic sequences. The perceptual hypothesis predicts that it should be harder to detect and identify a change on the longer interval following a series of short intervals. However, for all three rhythms, there were no significant main effects of position or interactions for both detections and identifications.

Melodic sequences. The perceptual hypothesis predicts that changes on the jumps (Positions 3 and 5) should be harder to detect and identify than changes in other positions. The percentage of detections for increases and decreases for the melodic jumps are shown in Figure 5. There were no main effects of position, but there was a significant interaction between position and direction of change $[F(5,40)=10.2, p<.01]$. However, the observed pattern did not correspond to the predictions. Instead, increases on the higher pitch events (Positions 3 and 4) were harder to detect than were increases in other positions, whereas the opposite pattern was seen for the decreases. Similar patterns were seen for the identifications. No changes in sensitivity were seen in relation to the melodic turns.

\section{Discussion}

The results of Experiment 2 do not provide support for the perceptual hypothesis since no systematic variations in the ability to detect and identify the position of intensity changes was observed as a function of the physical characteristics of the sequence.

\section{GENERAL DISCUSSION}

How can variations observed in the performance of short musical sequences be explained? The musical expression hypothesis, which suggests that variations are related to the musician's desire to transmit a particular musical interpretation to the listener, does not appear to apply in this case: variations do not entirely disappear when the musician is asked to play in a mechanical fashion. We have presented an alternative perceptual hypothesis whereby, in some musical sequences, some intervals are played longer/louder than others because these intervals are in fact perceived as shorter/softer than the others, and musicians compensate for this distortion by playing the intervals longer or louder.

The two experiments presented here were designed to test the validity of this perceptual hypothesis: the existence of systematic variations in the ability to detect and identify temporal and intensity variations would provide support for the perceptual hypothesis. Such variations in the sensitivity to changes in intensity were not seen, but corresponding changes were observed for the timing variations. Support for the perceptual hypothesis is therefore obtained for the timing variations, but not for the intensity variations.

\section{REFERENCES}

Clarke, E. F. (1988). Generative principles in music performance. In J. A. Sloboda (Ed.), Generative processes in music: The psychology of performance, improvisation and composition (pp. 1-25). Oxford: Oxford University Press.

DraKe, C. (1990). Processus cognitifs impliqués dans l'organisation du rythme musical. Unpublished doctoral dissertation, University of Paris $V$, France.

Drake, C., \& Palmer, C. (in press). Accent structures in music performance. Music Perception.

HalPERN, A. R., \& DARWIN, C. J. (1982). Duration discrimination in a series of rhythmic events. Perception \& Psychophysics, 31, 86-89.

Hirsh, I. J., Monahan, C. B., Grant, K. W., \& Singh, P. G. (1990). Studies in auditory timing: 1 . Simple patterns. Perception \& Psychophysics, 47, 215-226.

Monahan, C. B., \& HiRsh, I. J. (1990). Studies in auditory timing 2. Rhythm patterns. Perception \& Psychophysics, 47, 227-242.

Palmer, C. (1989). Mapping musical thought to musical performance. Journal of Experimental Psychology: Human Perception \& Performance, 15, 331-346.

Seashore, C. E. (1938). Psychology of music. New York: McGraw-Hill. Shaffer, L. H., Clarke, E. F., \& Todd, N. P. (1985). Meter and rhythm in piano playing. Cognition, 20, 61-77.

SLOBODA, J. A. (1983). The communication of musical meter in piano performance. Quarterly Journal of Experimental Psychology, 35A, 377-396.

TodD, N. (1985). A model of expressive timing in tonal music. Music Perception, 3, 635-641.

(Manuscript received September 23, 1992.) 